sumably, despite high local concentrations of stimulators in the tumor microenvironment, such as VEGF. How might this happen? Watanabe et al. (13) provide a potential answer on the basis of an experiment in which hypoxia - which is commonly associated with solid tumors - was found to suppress the VEGF-mediated induction of vasohibin in cultured endothelial cells. What about the effects on vasohibin by other endothelial cell-reactive inhibitors such as TSP-1? Might they actually suppress its expression, possibly leading to a form of induced or acquired resistance to various endogenous angiogenesis inhibitors (20-22)? Finally, if vasohibin acts on mature vascular endothelial cells, what are its effects on putative circulating peripheral blood endothelial progenitor cells (23)? In this regard, Watanabe et al. report that despite the lack of a classic secretion signal sequence, a cleaved form of vasohibin is apparently released from endothelial cells. This means it may freely circulate and, provided that sufficient concentrations can be attained in the blood, it could potentially affect either circulating endothelial cells or their progenitor subset cells, assuming such cells actually express a vasohibin receptor or binding element.

Clearly, these questions are only the tip of the vasohibin iceberg. As answers begin to unfold we shall learn whether or not an exciting new field in endothelial cell biology and angiogenesis has just been launched.
Address correspondence to: Robert S. Kerbel, Sunnybrook and Women's College Health Sciences Centre and the University of Toronto, S-217 Research Building, 2075 Bayview Avenue, Toronto, Ontario M4N 3M5, Canada. Phone: (416) 480-5711; Fax: (416) 4805884; E-mail: robert.kerbel@swchsc.on.ca.

1. Kerbel, R.S., and Folkman, J. 2002. Clinical translation of angiogenesis inhibitors. Nat. Rev. Cancer. 2:727-739.

2. Ferrara, N., Hillan, K.J., Gerber, H.P., and Novotny, W. 2004. Discovery and development of bevacizumab, an anti-VEGF antibody for treating cancer. Nat. Rev. Drug Discov. 3:391-400.

3. Dameron, K.M., Volpert, O.V., Tainsky, M.A., and Bouck, N. 1994. Control of angiogenesis in fibroblasts by p53 regulation of thrombospondin-1. Science. 265:1582-1584.

4. Kalluri, R. 2002. Discovery of type IV collagen noncollagenous domains as novel integrin ligands and endogenous inhibitors of angiogenesis. Cold Spring Harb. Symp. Quant. Biol. 67:255-266.

5. Lindner, D.J. 2002. Interferons as antiangiogenic agents. Curr. Oncol. Rep. 4:510-514.

6. Hanahan, D., and Folkman, J. 1996. Patterns and emerging mechanisms of the angiogenic switch during tumorigenesis. Cell. 86:353-364.

7. Sage, H. 1999. Pieces of eight: bioactive fragments of extracellular proteins as regulators of angiogenesis. Trends Cell Biol. 7:182-186.

8. Langer, R., Brem, H., Falterman, K., Klein, M., and Folkman, J. 1976. Isolations of a cartilage factor that inhibits tumor neovascularization. Science. 193:70-72.

9. Langer, R., Conn, H., Vacanti, J., Haudenschild, C., and Folkman, J. 1980. Control of tumor growth in animals by infusion of an angiogenesis inhibitor. Proc. Natl. Acad. Sci. U. S. A. 77:4331-4335.

10. Bouck, N., Stellmach, V., and Hsu, S.C. 1996. How tumors become angiogenic. Adv. Cancer Res. 69:135-174.

11. Folkman, J. 1985. Tumor angiogenesis. Adv. Cancer Res. 43:175-203.
12. Folkman, J. 1971. Tumor angiogenesis: therapeutic implications. N. Engl. J. Med. 285:1182-1186.

13. Watanabe, K., et al. 2004. Vasohibin as an endothelium-derived negative feedback regulator of angiogenesis. J. Clin. Invest. 114:898-907. doi:10.1172/ JCI200421152.

14. Hanahan, D., Bergers, G., and Bergsland, E. 2000. Less is more, regularly: metronomic dosing of cytotoxic drugs can target tumor angiogenesis in mice. J. Clin. Invest. 105:1045-1047.

15. Kerbel, R.S., and Kamen, B.A. 2004. Antiangiogenic basis of low-dose metronomic chemotherapy. Nat. Rev. Cancer. 4:423-436.

16. Bocci, G., Francia, G., Man, S., Lawler, J., and Kerbel, R.S. 2003. Thrombospondin-1, a mediator of the antiangiogenic effects of low-dose metronomic chemotherapy. Proc. Natl. Acad. Sci. U. S. A. 100:12917-12922.

17. Hamano, Y., et al. 2004. Thrombospondin-1 associated with tumor microenvironment contributes to low-dose cyclophosphamide-mediated endothelial cell apoptosis and tumor growth suppression. Cancer Res. 64:1570-1574.

18. Kalas, W., et al. 2003. Restoration of thrombospondin 1 expression in tumor cells harbouring mutant ras oncogene by treatment with low doses of doxycycline. Biochem. Biophys. Res. Commun. 310:109-114.

19. Volpert, O.V., et al. 2002. Inducer-stimulated Fas targets activated endothelium for destruction by anti-angiogenic thrombospondin-1 and pigment epithelium-derived factor. Nat. Med. 8:349-357.

20. Yu, J.L., Rak, J.W., Coomber, B.L., Hicklin, D.J., and Kerbel, R.S. 2002. Effect of p53 status on tumor response to antiangiogenic therapy. Science. 295:1526-1528.

21. Filleur, S., et al. 2001. In vivo mechanisms by which tumors producing thrombospondin 1 bypass its inhibitory effects. Genes Dev. 15:1373-1382.

22. Filleur, S., et al. 2003. SiRNA-mediated inhibition of vascular endothelial growth factor severely limits tumor resistance to antiangiogenic thrombospondin- 1 and slows tumor vascularization and growth. Cancer Res. 63:3919-3922.

23. Rafii, S., Lyden, D., Benezra, R., Hattori, K., and Heissig, B. 2002. Vascular and haematopoietic stem cells: novel targets for anti-angiogenesis therapy? Nat. Rev. Cancer. 2:826-835.

\title{
IRS2 takes center stage in the development of type 2 diabetes
}

\author{
Matthew J. Brady
}

Department of Medicine, Committee on Molecular Metabolism and Nutrition, University of Chicago, Chicago, Illinois, USA.

\begin{abstract}
The etiology of type 2 diabetes is characterized by obesity, insulin and leptin resistance, and compensatory $\beta$ cell hyperplasia followed by islet degeneration, resulting in the eventual dysregulation of glucose and lipid homeostasis. The recent identification of insulin receptor substrate 2 (IRS2) as a central player in the pathophysiology of many of these processes suggests a potentially unifying molecular link underlying the initiation and progression of type 2 diabetes (see the related articles beginning on pages 908 and 917).
\end{abstract}

Nonstandard abbreviations used: IRS, insulin receptor substrate.

Conflict of interest: The author has declared that no conflict of interest exists.

Citation for this article: J. Clin. Invest. 114:886-888 (2004). doi:10.1172/JCI200423108.
Diabetes mellitus is characterized by an inability of the body to efficiently utilize circulating nutrients. Normally, in response to elevation of plasma glucose, insulin is secreted by $\beta$ cells in the islets of Langerhans and promotes energy uptake, metabolism, and storage. Type 2 diabetes is preceded by the development of insulin resistance in target tissues (1), but the molecular causes of its initiation and progression remain unclear. Obesity is the predominant risk factor for the onset of insulin resistance, although other genetic and environmental factors also contribute (2). Islets initially compensate for the loss of insulin sensitivity by expanding $\beta$ cell mass and insulin secretory capacity, but over time, they cannot continue to meet the chronic stress of increased insulin demand. The loss of $\beta$ cells and reduction in circulating insulin levels occur 


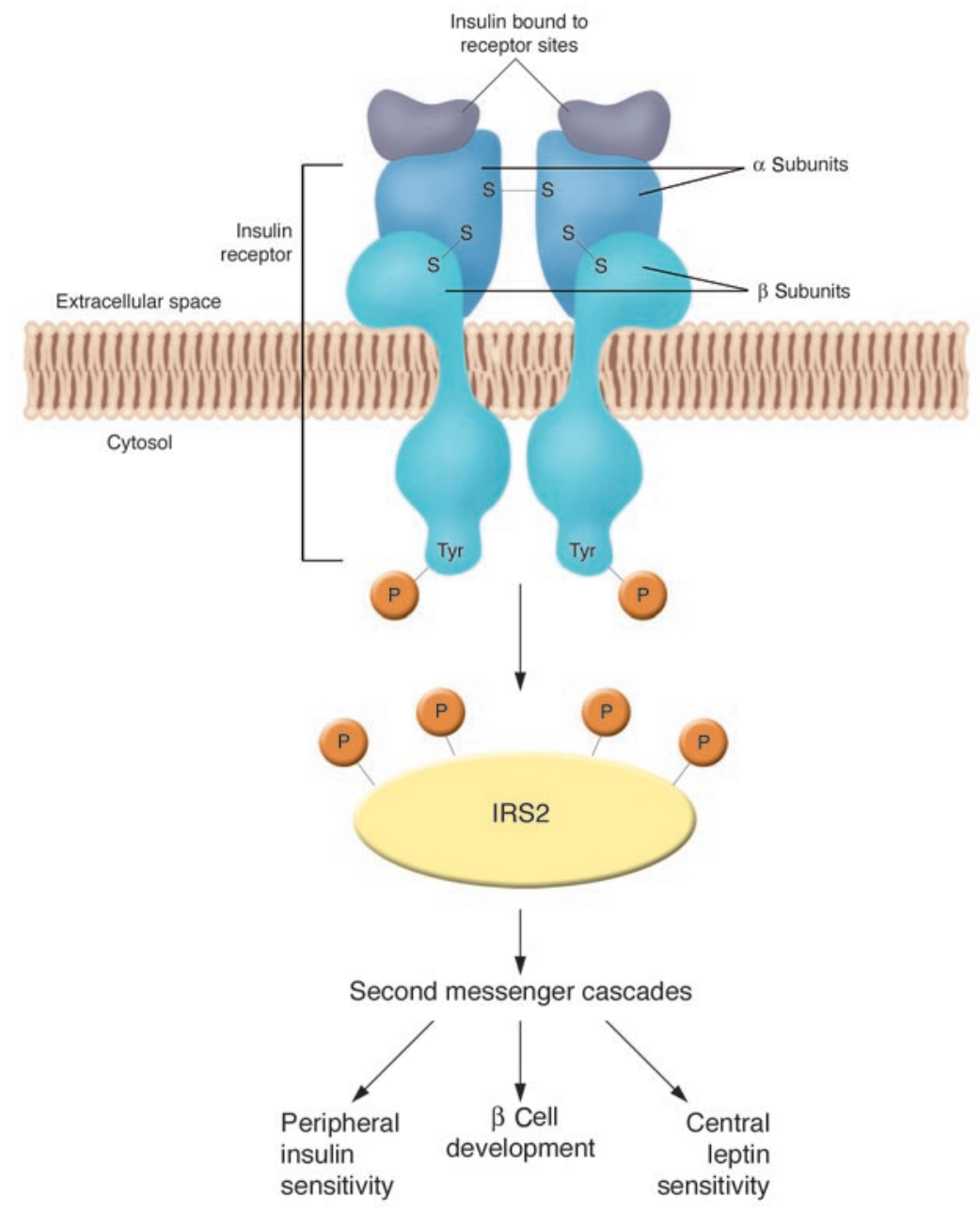

despite continuing peripheral insulin resistance, resulting in chronic hyperglycemia. However, the underlying molecular links among the cascade of obesity, insulin resistance, islet compensation, eventual $\beta$ cell failure, and onset of frank diabetes remain unidentified.

\section{IRS2 mediates insulin signaling in responsive cells}

Insulin signals by binding to a specific tyrosine kinase receptor expressed in target cells, which results in the increased phosphorylation of a number of substrate proteins and activation of complex second messenger cascades that regulate glucose and lipid metabolism (3). Insulin receptor substrate (IRS) proteins are a family of molecules that are directly phosphorylated by the insulin receptor (Figure 1), which leads to the recruitment and activation of additional signaling proteins (4). The crucial role of IRS proteins in insulin action has been demonstrated using transgenic knockout mouse models (5). Ablation of
Irs1 resulted in growth retardation and insulin resistance, but not overt diabetes due to increased $\beta$ cell mass (6-8). In contrast, Irs $2^{-/-}$mice displayed many of the hallmarks of type 2 diabetes in human subjects: peripheral insulin resistance coupled with a lack of compensatory $\beta$ cell expansion, resulting in hyperglycemia, diabetes, and premature death $(9,10)$. These results demonstrated a crucial role for IRS2 in insulin sensitivity in liver and skeletal muscle, as well as $\beta$ cell proliferation.

However, due to the systemic knockout of Irs 2 in these animals, the relative contribution of reduced IRS2 levels in various cell types to the development of diabetes remained unclear. In this issue of the JCI, Lin et al. (11) and Kubota et al. (12) independently generated a novel transgenic mouse model by crossing a floxed Irs 2 mouse with a mouse expressing Cre recombinase under the control of the rat insulin promoter. Thus, the floxed Irs 2 would only be excised from the genomic DNA in cell types that also expressed Cre, resulting in a tissue-spe-

\section{Figure 1}

Role of IRS2 in insulin signaling. Insulin binds to its receptor in target cells, leading to the activation of an intrinsic tyrosine kinase located in the $\beta$ subunit. The resulting autophosphorylation $(P)$ of the insulin receptor on tyrosine (Tyr) residues increases the association of various signaling molecules, including IRS2. Insulin receptor directly phosphorylates IRS2 on multiple tyrosine residues, which, in turn, initiates a variety of second messenger cascades. Recent work has demonstrated a critical role for IRS2 in the maintenance of peripheral insulin sensitivity, central leptin sensitivity, and proper $\beta$ cell development in the islets of Langerhans $(11,12)$. Thus, IRS2 plays a central role in preserving insulin action in multiple cell types, while reduction of IRS expression and/or function may be a fundamental cause of the development of insulin resistance, obesity, $\beta$ cell failure, and type 2 diabetes. S-S, disulphide bond. cific knockout of Irs 2 . In the offspring of these mice, Cre expression was, as expected, highest in the insulin-producing $\beta$ cells, but it also was present in several regions of the brain including the hypothalamus. The mice exhibited targeted disruption of Irs2, with levels reduced by greater than $90 \%$ in islets and $30-50 \%$ in the hypothalamus but no change in skeletal muscle, liver, or adipose tissue. Despite the restricted reduction in Irs2, these mice exhibited many of the same phenotypic changes seen in the global Irs $2^{-/-}$mice: central leptin resistance, peripheral insulin resistance, obesity, glucose intolerance, $\beta$ cell failure, and hyperglycemia. Of particular interest, partial reduction of hypothalamic Irs2 affected peripheral insulin sensitivity independently of reduced Irs2 levels in liver, muscle, or fat. Perhaps most unexpectedly, Lin et al. also reported the spontaneous reversal of diabetes in their transgenic mice upon aging due to $\beta$ cell regeneration and subsequent compensation for the persistent peripheral insulin resistance. 


\section{Targeted disruption of Irs2 expression results in multiple type 2 diabetic phenotypes}

Several intriguing phenotypes exhibited by the mice were described in these two reports $(11,12)$, although there were some unexplained temporal variations in weight gain and the appearance of hyperglycemia. Initially, the mice developed normally, but at four weeks of age, they began to display leptin resistance, hyperphagia, obesity, and insulin resistance. Interestingly, the development of peripheral insulin resistance could be uncoupled from increased fat mass, suggesting that the reduction in hypothalamic IRS2-mediated signaling affected insulin sensitivity in other tissues. Further complicating this issue was the development of leptin resistance in the transgenic animals, which was not explained by the obese phenotype and resulting elevation in circulating leptin levels. Several subtypes of hypothalamic neurons exert profound effects on feeding behavior (13). There is also evidence for crosstalk between leptin and insulin signaling that regulates hypothalamic neuronal energy sensing and appetite (14). However, the mechanisms by which reduced Irs2 expression in the brain diminishes both central leptin sensitivity and peripheral insulin sensitivity are unclear. Moreover, the precise identity of the neurons mediating these effects is not known, and indeed, their putative localization in the hypothalamus is a logical but unproven assumption. Additional work will be needed to untangle these complex and fascinating issues.

Despite a nearly complete lack of Irs2 in the $\beta$ cells, both groups reported normal islet development and size in young animals but a failure of islet mass to increase after 4 weeks $(11,12)$. Thus, no $\beta$ cell expansion occurred either in response to cues from normal animal growth or to compensate for the developing insulin resistance. These results alone would strongly suggest a critical role for IRS2 in postnatal $\beta$ cell proliferation. However, Lin et al. made the surprising discovery that as animals passed 6 months of age, the islets were substantially repopulated with new $\beta$ cells containing the floxed Irs 2 but inexplicably lacking Cre expression. Unfortunately, Kubota et al. did not study longer time points, so these results were not confirmed. Regardless of the mechanism mediating the loss of Cre expression, the fact that $\beta$ cell regeneration occurred in an adult diabetic animal model - and was substantial enough to correct the fasting hyperglycemia - is tremendously exciting. The identity of regenerating $\beta$ cells in adult subjects is controversial, with both progenitor cells and division of mature $\beta$ cells proposed as replenishing sources $(15,16)$. Given the explosion in the number of diabetic patients requiring islet transplantations, and the limited availability of donor tissue, the successful isolation of $\beta$ cell precursors would be a milestone in diabetes research. Unfortunately, the origin of the regenerated $\beta$ cells could not be identified in the present study and thus offers no resolution to the continuing debate on $\beta$ cell propagation. However, this work strongly suggests that enhancement of $\beta$ cell IRS2 expression in insulin-resistant patients might significantly delay islet destruction and onset of diabetes.

Together, these reports make a compelling argument for the central role of IRS2 in the hypothalamic regulation of central leptin and peripheral insulin sensitivity, as well as $\beta$ cell propagation. Investigation into the molecular mechanisms by which IRS2 mediates these disparate effects will undoubtedly increase our understanding of the initiation and development of type 2 diabetes.
Address correspondence to: Matthew J. Brady, University of Chicago, Department of Medicine, MC 1027, 5841 S. Maryland Avenue, Chicago, Illinois 60637, USA. Phone: (773) 702-2346; Fax: (773) 834-0486; E-mail: mbrady@medicine.bsd.uchicago.edu.

1. Shulman, G.I. 2000. Cellular mechanisms of insulin resistance. J. Clin. Invest. 106:171-176.

2. Saltiel, A.R. 2001. New perspectives into the molecular pathogenesis and treatment of type 2 diabetes. Cell. 104:517-529.

3. Saltiel, A.R., and Kahn, C.R. 2001. Insulin signalling and the regulation of glucose and lipid metabolism. Nature. 414:799-806.

4. White, M.F. 2002. IRS proteins and the common path to diabetes. Am. J. Physiol. Endocrinol. Metab. 283:E413-E422.

5. Nandi, A., Kitamura, Y., Kahn, C.R., and Accili, D. 2004. Mouse models of insulin resistance. Physiol. Rev. 84:623-647.

6. Tamemoto, H., et al. 1994. Insulin resistance and growth retardation in mice lacking insulin receptor substrate-1. Nature. 372:182-186.

7. Araki, E., et al. 1994. Alternative pathway of insulin signalling in mice with targeted disruption of the IRS-1 gene. Nature. 372:186-190.

8. Terauchi, Y., et al. 1997. Development of noninsulin-dependent diabetes mellitus in the double knockout mice with disruption of insulin receptor substrate-1 and beta cell glucokinase genes. Genetic reconstitution of diabetes as a polygenic disease. J. Clin. Invest. 99:861-866.

9. Withers, D.J., et al. 1998. Disruption of IRS-2 causes type 2 diabetes in mice. Nature. 391:900-904.

10. Kubota, N., et al. 2000. Disruption of insulin receptor substrate 2 causes type 2 diabetes because of liver insulin resistance and lack of compensatory beta-cell hyperplasia. Diabetes. 49:1880-1889.

11. Lin, X., et al. 2004. Dysregulation of insulin receptor substrate 2 in $\beta$ cells and brain causes obesity and diabetes. J. Clin. Invest. 114:908-916. doi:10.1172/JCI200422217.

12. Kubota, N., et al. 2004. Insulin receptor substrate 2 plays a crucial role in $\beta$ cells and the hypothalamus. J. Clin. Invest. 114:917-927. doi:10.1172/ JCI200421484.

13. Bjorbaek, C., and Kahn, B.B. 2004. Leptin signaling in the central nervous system and the periphery. Recent Prog. Horm. Res. 59:305-331.

14. Benoit, S.C., Clegg, D.J., Seeley, R.J., and Woods, S.C. 2004. Insulin and leptin as adiposity signals. Recent Prog. Horm. Res. 59:267-285.

15. Bonner-Weir, S., and Sharma, A. 2002. Pancreatic stem cells. J. Pathol. 197:519-526.

16. Dor, Y., Brown, J., Martinez, O.I., and Melton, D.A. 2004. Adult pancreatic beta-cells are formed by self-duplication rather than stem-cell differentiation. Nature. 429:41-46. 